\title{
Tratamiento Ortopédico de las Fracturas de Cóndilo Mandibular A Propósito de Tres Casos
}

\author{
Orthopedic Treatment for Mandibular Condyle Fracture: A Three Case Series
}

\author{
Salvador Valladares Pérez ${ }^{1,2}$; Diego Bustamante Correa ${ }^{1}$ \& Gerson Sepúlveda Troncoso ${ }^{1}$
}

\begin{abstract}
VALLADARES, S. P.; BUSTAMANTE, D. C. \& SEPÚLVEDA, G. T. Tratamiento ortopédico de las fracturas de cóndilo mandibular: A propósito de tres casos Int. J. Odontostomat., 13(2):157-161, 2019.

RESUMEN: Las fracturas de cóndilo mandibular son frecuentes en el trauma maxilofacial. Pueden tratarse ortopédicamente o en forma quirúrgica, dependiendo principalmente de las características morfológicas y funcionales del caso, y también de la experiencia del cirujano y los recursos disponibles. En los últimos años se ha establecido una corriente proclive al tratamiento quirúrgico, basada en la rápida recuperación del enfermo y la disminución de las secuelas funcionales y estéticas. Sin embargo, el tratamiento ortopédico bien indicado y ejecutado, puede ser una buena alternativa de tratamiento y que debe ser considerada dentro de las propuestas terapéuticas. El Objetivo de este estudio, es presentar una serie de tres casos de pacientes afectados por fracturas de cóndilo mandibular, tratados en forma ortopédica, en el Hospital El Carmen Dr. Luis Valentín Ferrada, Santiago - Chile. Además, se presentará una revisión de las dos tendencias de tratamiento con énfasis en las indicaciones y protocolo adecuado de tratamiento ortopédico.
\end{abstract}

PALABRAS CLAVE: cóndilo de la mandíbula, fractura, tratamiento.

\section{INTRODUCCIÓN}

Las fracturas que afectan al cóndilo mandibular son frecuentes en el trauma mandibular (Loukota et al., 2010). Su tratamiento ha seguido una tendencia clara en los últimos años hacia el tratamiento quirúrgico o abierto. Se han establecido en general dos tipos de tratamiento: La reducción quirúrgica con fijación rígida interna, y el tratamiento ortopédico (Khelemsky et al., 2016).

El tratamiento ortopédico busca recuperar la función articular mediante una inmovilización temporal de la función articular (mediante bloqueo intermaxilar elástico o alámbrico) para luego recuperar la funcionalidad y dinámica mediante ejercicios kinésicos, mientras que el tratamiento quirúrgico busca reducir los cabos fracturarios mediante el uso de placas y tornillos de osteosíntesis.

Ambos tratamientos comparten la mayoría de las complicaciones asociadas como son la mal oclusión, la alteración en la dinámica mandibular y anqui- losis témporo-mandibular, entre otras. Siendo la anquilosis la complicación más severa y compleja de tratar (Singh et al., 2010).

Cualquiera sea el método empleado para devolver la función articular, ambos son influidos por el tiempo transcurrido entre el trauma y la instauración del tratamiento, por las características individuales del paciente, que generan cambios en el pronóstico y rehabilitación final del enfermo (Singh et al.).

Los objetivos de tratamiento, independientemente de que tipo sea, busca la restauración de la función articular, manteniendo una articulación temporomandibular (ATM) asintomática con un adecuado rango de movilidad y una oclusión estable.

Tratamiento Ortopédico: El tratamiento ortopédico kinésico, se indica para aquellos casos en que la fractura posee poco desplazamiento, estabilidad oclusal, mínima alteración en la dimensión vertical y se basa

\footnotetext{
${ }^{1}$ Hospital El Carmen Dr. Luis Valentín Ferrada, Maipú, Santiago, Chile.

${ }^{2}$ Escuela de Odontología Pontificia Universidad Católica, Santiago, Chile.
} 
en el re-establecimiento de la oclusión dentaria mediante bloqueo intermaxilar elástico o alámbrico y luego, ejercicios de movilidad articular. No existe un consenso ni se encuentra protocolizado en la literatura el tiempo y forma de la terapia elástica, ni la pauta de ejercicios de dinámica mandibular de éste (Rozeboom et al., 2017). Según una revisión sistemática de 2017 (Rozeboom et al.), se concluye que se requiere un período de bloqueo elástico y una rápida movilización de la articulación para evitar la anquilosis témporo mandibular. Según lo descrito por Rozeboom et al., el esquema de ejercicios más utilizado corresponde al bloqueo intermaxilar de 1 semana con dieta papilla. Posterior a esto, un bloqueo de 2 a 6 semanas intermitente con dieta blanda, acompañado de series de ejercicios de dinámica mandibular (apertura-cierre y lateralidades). Posterior a esto, un período de 2 a 6 semanas con bloqueo elástico intermitente diurno, ejercicios de dinámica mandibular y dieta blanda. El tiempo total de tratamiento varía según la evolución de cada caso. Cabe mencionar que en dicho meta-análisis, no se considera el bloqueo alámbrico en ningún estudio reciente considerado en la revisión (Rozeboom et al.).

Tratamiento quirúrgico: El tratamiento quirúrgico se ha descrito para aquellos casos en que el cóndilo se encuentra luxado más de $45^{\circ}$ en sentido latero-medial, con pérdida de la dimensión vertical posterior y alteraciones oclusales secundarias. Por otro lado, la orientación del rasgo de fractura y el tamaño de los fragmentos óseos debe permitir la colocación de elementos de osteosíntesis con al menos dos tornillos a cada lado del plano fracturario. Se utilizan abordajes preauriculares, retromandibulares, sub mandibular extendido o combinación de estos, existiendo complicaciones asociadas inherentes a éstos (parálisis facial transitoria o permanente, fistula salival, infección de la herida, entre otros). Por otro lado, en el postoperatorio inmediato y mediato se requiere, en muchos casos, de terapia elástica y ejercicios de di-

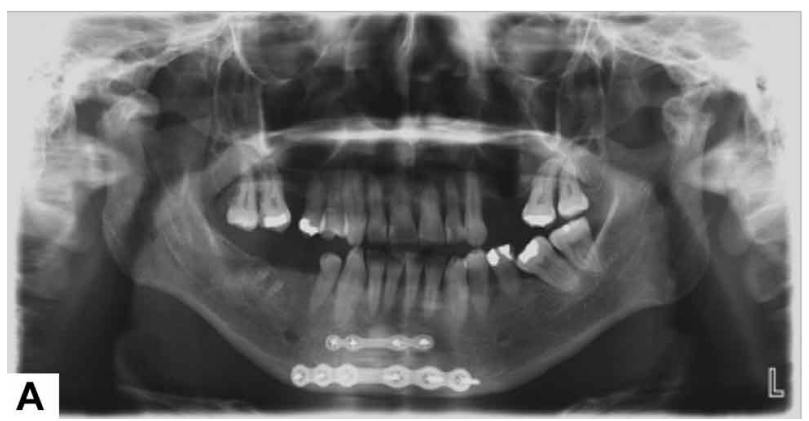

námica mandibular similares al tratamiento ortopédicokinésico (Singh et al.).

Presentación de los casos: A continuación, se presentan una serie de tres casos tratados mediante tratamiento ortopédico en el Hospital El Carmen Dr. Luis Valentín Ferrada, Santiago.Para los tres pacientes se realizó el mismo protocolo de tratamiento el cual consiste en realizar en primera instancia estabilización mediante bloqueo intermaxilar con elásticos por un período de 5 días con buena higiene y dieta papilla. Posterior a esto, se realiza un bloqueo de manera intermitente durante un período de 10 a 15 días, utilizado en forma continua durante la noche e intermitente durante el día. En esta fase durante el día los retira para realizar aseo oral, ejercicios y alimentarse mediante dieta papilla o blanda. Los ejercicios corresponden a 6 series de movimientos de apertura y cierre, lateralidad y protrusiva. Las series se realizan 4 veces al día repitiendo 5 veces cada serie. La tercera fase del tratamiento, consiste en la utilización de los elásticos solamente durante la noche y continuar con el mismo patrón de ejercicios y dieta durante el día hasta finalmente completar 3 meses desde el trauma.

Caso A. hombre 56 años de edad sin antecedentes mórbidos ni alergias conocidas. Sufre caída a nivel durante su trayecto laboral causando fractura parasinfisiaria mandibular derecha y cuello de cóndilo bilateral. El paciente fue atendido en primera instancia en un centro hospitalario de la seguridad laboral, realizándose reducción y osteosíntesis de la fractura parasinfisiaria. Cinco semanas posterior al accidente, fue derivado a nuestro servicio para continuar su tratamiento. Al examen clínico, se observa alteración en la dinámica mandibular, alteración oclusal con mordida abierta anterior por tope molar posterior, desdentamiento parcial, dimensión vertical conservada sin evidencia de dolor articular, rangos de apertura oral de $30 \mathrm{~mm}$ interincisal.

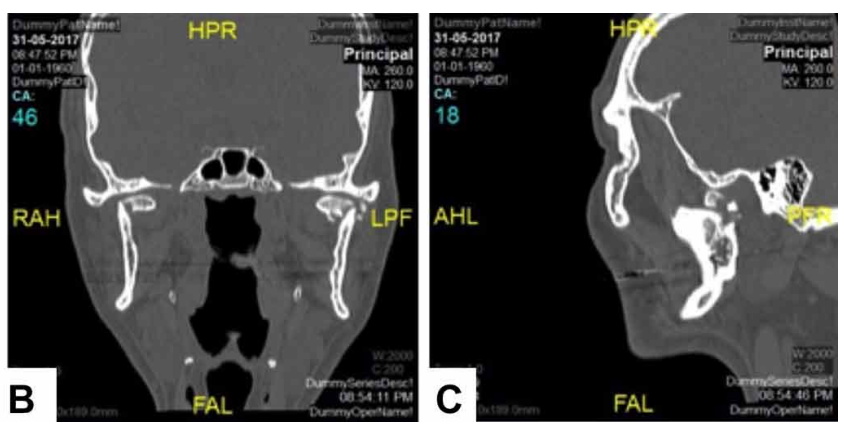

Fig. 1. A. Radiografía panorámica se observa fractura bilateral del cuello del cóndilo; 1. B, C. Tomografía se observa fractura de cóndilo bilateral tipo V según Spiessl and Schroll. 
A la radiografía panorámica (Fig. $1 \mathrm{~A})$, se evidencia una fractura bilateral del cuello del cóndilo, y en la tomografía (Fig. 1B,C), se observa fractura de cóndilo bilateral tipo $\mathrm{V}$ según Spiessl and Schroll (Powers, 2017). Debido al tiempo transcurrido desde la fractura, se instaura tratamiento ortopédico para devolver la correcta función articular y una oclusión estable, mediante terapia ortopédica según protocolo previamente descrito. El paciente evolucionó de manera favorable sin sintomatología articular, una dimensión vertical sin alteración, una apertura oral dentro de rangos mayores a $40 \mathrm{~mm}$, movimientos con escasa limitación y una oclusión estable sin mordida abierta anterior. El paciente completó un año de seguimiento sin complicaciones, manteniendo controles periódicos en nuestro centro.

Caso B: Hombre 27 años de edad sin antecedentes mórbidos ni alergias conocidas que sufre golpe durante práctica deportiva en la región mentoniana causándole una fractura mandibular paramediana izquierda y cuello de cóndilo izquierdo. Al examen clínico, paciente desdentado parcial, estabilidad oclusal, dimensión vertical conservada, limitación en la apertura y lateralidades. A la radiografía panorámica (Fig. 2A) y tomografía (Fig. 2B,C), se evidencia una fractura de cuello de cóndilo tipo III según Spiessl and Schroll (Powers). Se le realiza reducción y osteosíntesis e instalación de 6 micro tornillos (sistema IMF Synthes), tres superiores y tres inferiores, en línea media y en las zonas laterales para realizar bloqueo elástico por un período de 15 días y posteriormente uso de manera intermitente y serie de ejercicios de apertura, cierre, lateralidades y protusivas descrito anteriormente por período de 3 meses. El paciente evolucionó de manera favorable sin sintomatología articular, una dimensión vertical sin alteración, una apertura bucal dentro de rangos mayores a $40 \mathrm{~mm}$, movimientos con escasa limitación y una oclusión estable sin mordida abierta anterior. El paciente completó un año de seguimiento sin complicaciones, manteniendo controles periódicos en nuestro centro.

Caso C: Hombre 18 años de edad sin antecedentes mórbidos ni alergias conocidas, sufre golpe en región facial y sub mental durante práctica deportiva. Acude al servicio de urgencia al Hospital el Carmen debido a alteración oclusal, limitación en la apertura mandibular y lateralidades. Al examen físico, paciente con erosiones faciales, dolor en región pre-auricular. No presenta aumentos de volúmenes a nivel facial, pero encontramos usencia de cóndilo izquierdo a la palpación de este. Presenta limitación en la apertura, lateralidades y protusiva, dentición completa, inestabilidad oclusal y dimensión vertical conservada. En la radiografía panorámica (Fig. 3A) y tomografía computarizada (Fig. $3 B, C)$, se evidencia una fractura de cuello de cóndilo izquierdo tipo II según Spiessl and Schroll (Powers)
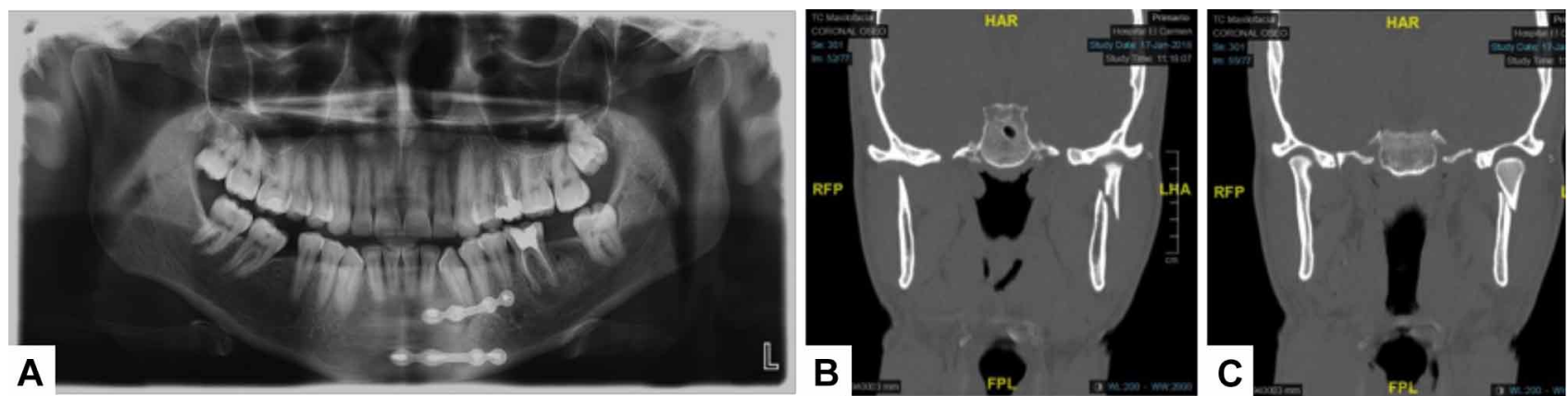

Fig. 2. A.Radiografía panorámica; Fig. 2B, C. Tomografía, se observa fractura de cuello de cóndilo tipo III, según Spiessl and Schroll (Powers).
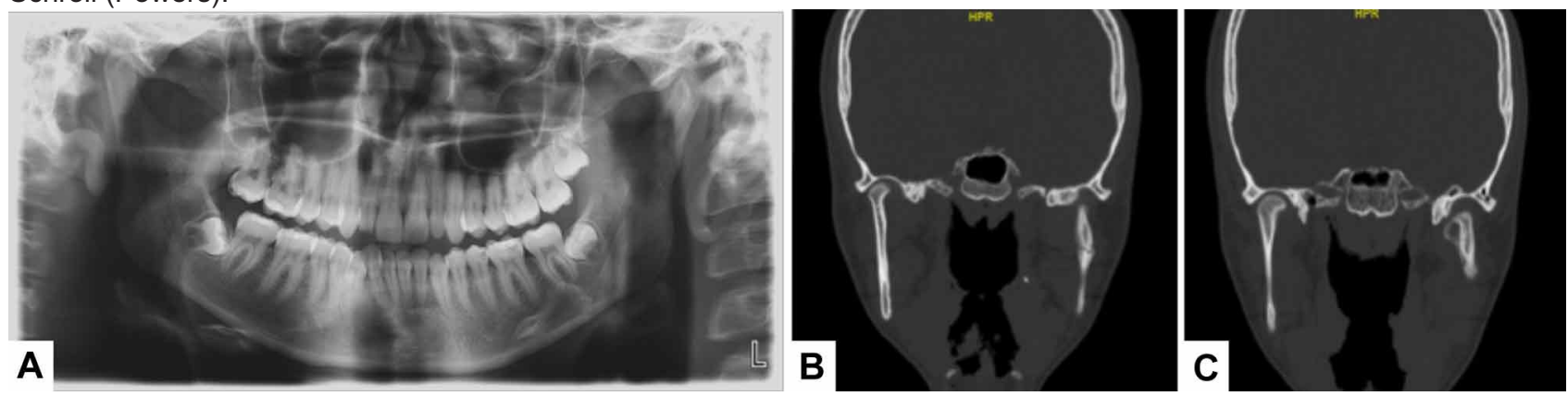

Fig. 3. A. Radiografía panorámica; Fig. 3B, C. Tomografía computarizada, se observa fractura de cuello de cóndilo izquierdo tipo Il según Spiessl and Schroll (Powers). 
con poco desplazamiento. Se realizó estabilización y bloqueo intermaxilar elástico y se decidió realizar un tratamiento de tipo ortopédico debido a la estabilidad de la oclusión y el poco desplazamiento de los cabos fracturados. Se mantuvo bloqueo elástico por un período de 10 días y posteriormente debido a evolución favorable, se comenzó con serie de ejercicios y uso de elásticos de manera intermitente por un período total de tres meses. Finalmente, el paciente evolucionó de manera favorable sin sintomatología articular, una dimensión vertical sin alteración, una apertura oral dentro de rangos mayores a $40 \mathrm{~mm}$, movimientos con escasa limitación y una oclusión estable. El paciente completó un año de seguimiento sin complicaciones, manteniendo controles periódicos en nuestro centro.

\section{DISCUSIÓN}

En base a las características de los casos previamente expuestos, el tratamiento Ortopédico parece ser una alternativa viable para el tratamiento de fractura de cóndilo siempre y cuando se cumpla ciertas indicaciones tales como; estabilidad oclusal, máxima inter-cuspidación dental, poseer remanente dentario, conservación de la dimensión vertical, fracturas con poco desplazamiento, fracturas no conminutadas y que fracturas concomitantes sean reducidas y estabilizadas (Sing et al 2010). Si bien los resultados de este tratamiento parecen ser favorables a corto plazo, se debe de realizar un seguimiento continuo de estos pacientes a largo plazo debido a que las complicaciones en este tipo de fracturas pueden expresarse hasta un período de 10 años (Chrcanovic et al., 2015).

Gracias a los avances tecnológicos y advenimiento de nuevos materiales de osteosíntesis, el tratamiento quirúrgico de las fracturas de cóndilo mandibular ha aumentado considerablemente en los últimos años (Singh et al.). Aún con lo señalado anteriormente, los tratamientos quirúrgicos no están exentos de complicaciones, pero si han demostrado una recuperación de la función articular más rápida que el tratamiento ortopédico (Singh et al.).

Según lo anteriormente expuesto, no existe evidencia científica concreta que demuestre que un tratamiento es mejor que el otro. Ambos tratamientos poseen sus respectivas ventajas y comparten las mismas complicaciones y riesgos por lo que los controles periódicos y continuos en estos pacientes es de vital importancia para poder interceptar a tiempo las posibles complicaciones (Chrcanovic et al.). Si bien el tratamiento quirúrgico nos brinda una recuperación más pronta de la dinámica mandibular, el tratamiento ortopédico es una opción más simple y fácil de realizar, evitándole al paciente ser sometido a una intervención quirúrgica y disminuir las complicaciones propias de la intervención como lo son las lesiones del nervio facial, infección de la herida operatoria, hemorragia, entre otros (Singh et al.; Chrcanovic et al.). Distintos estudios hacen comparación entre ambos tipos de tratamiento exponiendo las ventajas y desventajas de las distintas alternativas terapéuticas en donde todos concuerdan que la decisión de elegir uno u otro tratamiento dependerá de diversos factores siendo el desplazamiento del rasgo de fractura, el factor decidor si considerar un tratamiento ortopédico o abierto (Singh et al.; Chrcanovic et al.).

Finalmente, podríamos señalar que el tratamiento ortopédico es una alternativa válida como tratamiento definitivo para las fracturas de cóndilo cuando posee una correcta indicación. Es Importante recalcar que la terapia kinésica y la limitación de la función masticatoria, son vitales para el éxito en este tipo de tratamiento. (Chrcanovic et al.). Debido a que la recuperación de la función articular depende del mismo paciente, es de vital importancia para el éxito de éste, que el paciente esté comprometido con el tratamiento de manera efectiva. Si bien el tratamiento quirúrgico de las fracturas de cóndilo nos permite una mejor reducción de los rasgos de fractura y una recuperación más rápida de la función articular, esta no se logra si el paciente no cumple con el tratamiento kinésico y terapia elástica postoperatoria (Singh et al.; Chrcanovic et al.).

VALLADARES, S. P.; BUSTAMANTE, D. C. \& SEPÚLVEDA, G. T. Orthopedic treatment for mandibular condyle fracture: A three case series. Int. J. Odontostomat., 13(2):157-161, 2019.

ABSTRACT: The Mandible condyle fracture, are common in maxillofacial trauma. They can be treated orthopedically or surgically, depending on the characteristics of the case, the available means and the experience of the surgeon. In recent years, a trend has been established that is prone to surgical treatment, based on the patient's rapid recovery and the reduction of aesthetic and functional sequelae. However, orthopedic treatment can be an excellent treatment alternative. This is why today the treatment of mandibular condyle fractures has become a controversy in the specialty. The objective of this report is to present a series of three cases of patients with mandibular condylar frac- 
tures, treated orthopedically, at the Carmen Clinical Hospital, Santiago - Chile. In addition with a brief review of the two treatment trends will be presented with emphasis on the indications and appropriate protocol of orthopedic treatment. treatment.

KEY WORDS: Mandibular condyle fracture, REFERENCIAS BIBLIOGRÁFICAS

Chrcanovic, B. R. Surgical versus non-surgical treatment of mandibular condylar fractures: a meta-analysis. Int. J. Oral Maxillofac. Surg., 44(2):158-79, 2015.

Khelemsky, R.; Moubayed, S. P. \& Buchbinder, D. What is the evidence for open versus closed treatment of mandibular condylar fractures in adults? Laringoscope, 126(11):2423-5, 2016.

Loukota, R. A.; Neff, A. \& Rasse, M. Nomenclature/classification of fractures of the mandibular condylar head. Br. J. Oral Maxillofac. Surg., 48(6):477-8, 2010.

Powers, D. B. Classification of mandibular condylar fractures. Atlas Oral Maxillofac. Surg. Clin. North Am., 25(1):1-10, 2017.

Rozeboom, A. V. J.; Dubois, L.; Bos, R. R. M.; Spijker, R. \& de Lange, $\mathrm{J}$. Closed treatment of unilateral mandibular condyle fractures in adults: a systematic review. Int. J. Oral Maxillofac. Surg., 46(4):456-64, 2017.

Singh, V.; Bhagol, A.; Goel, M.; Kumar, I. \& Verma, A. Outcomes of open versus closed treatment of mandibular subcondylar fractures: a prospective randomized study. J. Oral Maxillofac. Surg., 68(6):1304-9, 2010.
Dirección para correspondencia:

Salvador Valladares Pérez

Hospital El Carmen Dr. Luis Valentín Ferrada

Santiago

Camino A Rinconada 1201

Maipú

Santiago

CHILE

Email: salvadorvalladares@gmail.com

Recibida : 01-08-2018

Aceptada: 21-01-2019 